\title{
The Effect of Atrial Fibrillation on Perioperative Outcomes in Patients With Pancreatic Cancer Undergoing Open Pancreaticoduodenectomy: Analysis of the National Inpatient Sample
}

\section{Pu Han}

Beijing Friendship Hospital

\section{Yifeng Yang}

University of lowa Hospitals and Clinics

Yanjie He

New York University Langone Medical Center

Hongwei Wu

Beijing Friendship Hospital

\section{Dong Wang}

Beijing Friendship Hospital

Kun Liu

Beijing Friendship Hospital

Chengjian Guan

Beijing Friendship Hospital

Xiaodong Zhang

Beijing Friendship Hospital

Wei Guo

Beijing Friendship Hospital

Zhongtao Zhang ( $\square$ zhangzht@ccmu.edu.cn )

Beijing Friendship Hospital

\section{Research Article}

Keywords: pancreatic cancer, pancreaticoduodenectomy, atrial fibrillation, complication

Posted Date: December 9th, 2020

DOl: https://doi.org/10.21203/rs.3.rs-116627/v1

License: (c) (1) This work is licensed under a Creative Commons Attribution 4.0 International License. Read Full License 
Page 2/16 


\section{Abstract}

Background: To assess the role of atrial fibrillation (AF) on perioperative outcomes in patients with pancreatic cancer undergoing open pancreaticoduodenectomy (OPD).

Methods: We investigated patients with pancreatic cancer undergoing OPD during 2012-2014 within National Inpatient Sample (NIS) database. The study population was divided into two groups based on the presence of AF. In-hospital mortality, length of stay (LOS), cost of hospitalization, and in-hospital complications were compared between the two groups. Logistic regression models and linear regression were used to adjust for potential confounders.

Results: Of the 12,785 patients aged $\geq 18$ years undergoing OPD during years $2012-2014,11,469$ (90\%) had no AF and 1,316 (10\%) had AF. Patients with AF were found to have significantly higher cost (coefficient $0.35,95 \% \mathrm{Cl} 0.06-0.64, \mathrm{P}=0.02$ ), but similar mortality (OR $3.57,95 \% \mathrm{Cl} 0.86-14.87, \mathrm{P}=0.08$ ) and LOS (coefficient $0.16,95 \% \mathrm{Cl} 0-0.33, \mathrm{P}=0.052$ ) compared to those without AF. The risk of gastrointestinal anastomotic leakage (OR 22.11, 95\% Cl 3.25-150.22, $p=0.002)$, cardiac complications (OR 17.88, 95\% Cl 5.32-60.11, $\mathrm{p}<0.001$ ), respiratory complications (OR 2.38, 95\% Cl 1.23-4.60, $\mathrm{p}=0.01$ ), pulmonary embolism (OR 18.53, 95\% $\mathrm{Cl} 2.67-128.62, \mathrm{p}=0.003$ ), and perioperative shock (OR $5.16,95 \% \mathrm{Cl}$ $1.03-25.72, p=0.045$ ) were found to be significantly higher in AF group than non-AF group. In elderly patients (> $65 \mathrm{y}$ ), AF was found to be associated with a significantly higher cost (coefficient $0.38,95 \% \mathrm{Cl}$ $0.05-0.71, P=0.023$ ), longer LOS (coefficient $0.21,95 \% \mathrm{Cl} 0.03-0.39, \mathrm{P}=0.023$ ), higher incidence of cardiac complications (OR 17.38, 95\% $\mathrm{Cl} 3.42-88.43, \mathrm{p}=0.001$ ), respiratory complications (OR 2.93, 95\% $\mathrm{Cl} 1.31-6.53, \mathrm{p}=0.009$ ) and postoperative shock (OR 27.06, 95\% $\mathrm{Cl} 2.35-311.15, \mathrm{p}=0.008$ ), yet similar mortality (OR 3.35, 95\% Cl 0.58-19.37, $\mathrm{P}=0.177)$.

Conclusions: AF was found to be associated with higher cost in pancreatic cancer patients undergoing OPD, as well as increased occurrence of cardiac complications, respiratory complications, pulmonary embolism and perioperative shock. Surgeons should pay special attention to these patients and formulate an appropriate perioperative evaluation plan.

\section{Background}

Pancreatic cancer is one of the most deadly neoplasm in the United States and it is the fourth leading cause of death from cancer[1]. A pancreaticoduodenectomy (PD), pancreatoduodenectomy, Whipple procedure, or Kausch-Whipple procedure is reference surgical option for the resection of pancreatic tumors[2]. During a PD, pancreatic head, common bile duct, gallbladder, and duodenum along with some part of the proximal jejunum need to be removed, and partial gastrectomy might be conducted[3]. The approach of PD includes open pancreaticoduodenectomy (OPD), laparoscopic pancreaticoduodenectomy (LPD) and robot-assisted pancreaticoduodenectomy (RPD).

Atrial fibrillation (AF) is the most common arrhythmia (1-2\% in the general population)[4], and it is associated with increased perioperative mortality and morbidity[5]. Several studies have proved that AF is 
related to bad outcomes and more perioperative complications[6, 7]. However, there is limited literature evaluating the impact of AF on outcomes of patients with pancreatic cancer undergoing OPD, especially through large-scale studies. The purpose of this study was to investigate the effect of AF on perioperative outcomes in patients with pancreatic cancer undergoing OPD.

\section{Methods}

\section{Data Source}

Supported by the Healthcare Cost and Utilization Project (HCUP), the National Inpatient Sample (NIS) is one of the largest inpatient administrative databases in the U.S. It represents approximately $20 \%$ of hospitalization in the USA, and it extracts data of nearly 8 million hospital discharges from more than 1,000 hospitals per year. The diagnosis and procedure codes in NIS database are based on the International Classification of Diseases, Ninth Revision, and Clinical Modification, Ninth Revision (ICD-9$\mathrm{CM}$ ). The NIS contains over 100 patient level data elements (demographic, diagnostic, and procedural) as well as hospital data such as location, median income of patients by zip code, teaching status, etc. Data from 2012 to 2014 were investigated. As NIS data are de-identified, this study was exempt from institutional review board evaluation.

\section{Study Population}

We searched for patient discharge data (ICD-9-CM) and hospital characteristics of patients $\geq 18$-year-old during years 2012-2014. The population of interest in this study was patients who underwent OPD, identified by ICD-9-CM procedure code 52.7. Robotic assisted procedures (ICD-9 procedure code 17.4) and laparoscopic procedures (ICD-9 procedure code 54.21) were excluded. Non-elective procedures were also excluded. Patients were then stratified into 2 groups based on the presence of AF (ICD-9-CM code 427.3). Patient demographics (age, sex, race, income, insurance status), hospital characteristics (location, teaching status and size) and common comorbidities were identified with ICD-9-CM codes (Supplement Table 1). Patients with missing data were excluded from our analyses.

\section{Study endpoints}

The primary endpoint of this study included (1) in-hospital mortality; (2) length of stay (LOS); (3) cost and (4) in-hospital complications, including gastrointestinal (GI) anastomotic leakage, GI bleeding, acute kidney injury (AKI), gastroparesis, blood transfusion, cardiac complications, respiratory complications, pneumonia, pulmonary embolism (PE), urinary tract infection (UTI), cardiac arrest, postoperative sepsis, postoperative shock and wound complications.

\section{Statistical Analysis}

We used a survey-specific method, with commands svyset and svy with pweight using DISCWT for 2012 to 2014 to generate nationwide estimates for each year. The stratum statement specifies NIS_STRATUM as the stratum identifier and the cluster statement specifies HOSP_NIS as the cluster identifier. 
Descriptive statistics was used to compare demographic characteristics and outcomes between OPD patients with and without AF. The baseline characteristics were compared between the AF group and control group using the Chi-square test for categorical variables and Student's t-test for continuous variables. In our multivariable regression model, we assessed differences in binary outcomes by using logistic regression (logistic command in Stata [StataCorp]) and in continuous outcomes by using linear regression (Stata command regress). All above were carried out in weighted samples.

Analyses were performed using Stata version 14 (StataCorp, College Station, TX). All statistical tests were two-sided, and $P$ values less than 0.05 were considered statistically significant.

\section{Results}

\section{Patient and hospital characteristics}

A total of 12,785 patients aged $\geq 18$ years underwent OPD during years 2012-2014 (Figure 1 ). Among them, 11,469 (90\%) had no AF and 1,316 (10\%) had AF. There were significantly more male, more Caucasian, older and Medicaid patients in AF group. There was no statistically significant difference in median annual income, hospital bed size, region, location and teaching status. As for comorbidities, history of myocardial infarction (MI), hypertension, diabetes mellitus, history of stroke, peripheral vascular disease (PVD), congestive heart failure (CHF), chronic kidney disease (CKD), chronic obstructive pulmonary disease (COPD), valvular heart disease (VHD), coronary artery disease (CAD) and cirrhosis were more prevalent in the AF group. There was no significant difference in thrombocytopenia, chronic liver disease and obesity between the AF and non-AF group (Table 1).

\section{Mortality}

The in-hospital mortality rate for all patients was 3.17\%. Mortality in AF group was higher than that in non-AF group (7.60\% versus $2.66 \%$ ). Multivariable regression analysis revealed that there is a trend that AF was associated with higher mortality in patients with pancreatic cancer undergoing OPD (adjusted OR $3.57,95 \% \mathrm{Cl} 0.86-14.87, \mathrm{P}=0.08$ ). We further focused on elder patients (age > 65). However, results of multivariable regression analysis still showed no significant difference (adjusted OR $3.35,95 \% \mathrm{Cl} 0.58$ 19.37, $\mathrm{P}=0.177)$ (Table 2, Figure 2).

\section{Length of stay}

The in-hospital mean LOS for all patients with OPD was 12.1 days. Unadjusted analysis showed patients with AF had longer LOS (14.6 days versus 11.8). However, after multivariate regression we didn't get significant result (adjusted coefficient $0.16,95 \% \mathrm{Cl} 0-0.33, \mathrm{P}=0.052$ ). Significant difference was found in the subgroup analysis in patients older than 65 years (adjusted coefficient $0.21,95 \% \mathrm{Cl} 0.03-0.39, \mathrm{P}=$ 0.023) (Table 2, Figure 2).

\section{Cost of hospitalization}


The in-hospital mean cost for all patients with OPD was 40,450.05 US dollars. Unadjusted analysis showed patients with AF had a significantly higher cost of hospitalization $(54,601.47$ dollars versus $38,812.80$ dollars). After multivariate regression we still got significant result (adjusted coefficient 0.35 , $95 \% \mathrm{Cl} 0.06-0.64, \mathrm{P}=0.02$ ). Among elder patients (> 65 years old), there was still significant difference between AF group and non-AF group on cost of hospitalization (adjusted coefficient $0.38,95 \% \mathrm{Cl} 0.05-$ $0.71, P=0.023$ ) (Table 2, Figure 2).

\section{In-hospital complications}

We also focused on in-hospital complications using univariate screening and multivariate regression. Patients in AF group has significantly higher incidence of $\mathrm{Gl}$ anastomotic leakage (OR $22.11,95 \% \mathrm{Cl} 3.25$ $150.22, p=0.002$ ), cardiac complications (OR 17.88, 95\% Cl 5.32-60.11, $p<0.001$ ), respiratory complications (OR 2.38, 95\% Cl 1.23-4.60, $p=0.01)$, PE (OR 18.53, 95\% Cl 2.67-128.62, $p=0.003)$, postoperative shock (OR $5.16,95 \% \mathrm{Cl} 1.03-25.72, \mathrm{p}=0.045)$ than non-AF group. While no difference in $\mathrm{GI}$ bleeding, AKI, gastroparesis, blood transfusion, pneumonia, UTI, cardiac arrest, postoperative sepsis and wound complications was observed. Among patients older than 65 years, significantly higher incidence of cardiac complications (OR $17.38,95 \% \mathrm{Cl} 3.42-88.43, \mathrm{p}=0.001$ ), respiratory complications (OR 2.93 , $95 \% \mathrm{Cl} 1.31-6.53, \mathrm{p}=0.009$ ) and postoperative shock (OR 27.06, 95\% $\mathrm{Cl} 2.35-311.15, \mathrm{p}=0.008$ ) was found in AF group (Table 2, Figure 2).

\section{Discussion}

PD is the reference standard surgical method for the treatment of tumors in the head of the pancreas, the lower end of the common bile duct, the duodenal papilla, and certain benign lesions. The current perioperative mortality of PD is less than $5 \%[8]$, but the incidence of perioperative complications is up to $27.1 \%$ or even higher[9]. The prognosis of PD is poor, and the 5-year survival rate after surgery is only $16 \%-20 \%[10,11]$. Common complications of PD include pancreatic fistula, bile leakage, $\mathrm{GI}$ anastomotic leakage, GI bleeding, AKI, gastroparesis, cardiac complications, respiratory complications, UTI, postoperative sepsis, postoperative shock, wound complications, etc.[12, 13] Previous studies have shown that perioperative complications have an adverse effect on survival and prognosis in various tumor types including esophageal cancer[14], gastric cancer[15] and colorectal cancer[16]. Similarly, in pancreatic cancer, the occurrence of perioperative complications also adversely affects survival and prognosis.

AF is the most common type of arrhythmia[4], accounting for $1-2 \%$ of the overall population. Patients with $\mathrm{AF}$ are at higher risk of thrombo-embolic disease and often require regular anticoagulation therapy[17]. It has been reported that AF can significantly promote the occurrence of perioperative complications of various surgical operations including urinary system surgery and liver transplantation, etc. $[6,7,18]$

To the best of our knowledge, this is the first population-based study that investigated the impact of AF on the outcomes of pancreatic cancer patients with OPD. The reports on mortality of PD varies in various 
studies. Cameron et al. summarized 1,000 consecutive PD and found that only 10 postoperative deaths, for a mortality of $1 \%[19]$. Kneuertz et al. reported that the 30-day mortality after PD was 2.9\%[20]. Our study found that during three-year time from 2012 to 2014, the overall in-hospital mortality was $3.17 \%$. The in-hospital mortality was $7.6 \%$ and $2.66 \%$ in the AF group and non-AF group, respectively, without significant difference (adjusted OR 3.57, 95\% Cl 0.86-14.87, P = 0.08). Kumar et al.[21] found that pulmonary complications were the leading cause for mortality after PD. Other causes of death include bile sepsis, liver failure, MI, pancreatic leak, etc. Narayanan et al.[22] showed that the most common cause of death after PD within 90 days is multisystem organ failure, followed by post-pancreatectomy hemorrhage, and cardiopulmonary arrest from myocardial infarction or pulmonary embolus. LOS was longer in AF group, but no significant difference was found (14.6 days versus 11.8, adjusted coefficient $0.16(0-0.33), P=0.052)$. There was a significant difference in cost between the two groups (54601.47 dollars versus 38812.80 dollars, adjusted coefficient $0.35(0.06-0.64), P=0.02)$. The need of more thorough evaluation of cardiac function and more complex management of perioperative complications might be a possible reason.

Our study found that the AF group had more perioperative complications than the non-AF group, including $\mathrm{Gl}$ anastomotic leakage, cardiac complications, respiratory complications, $\mathrm{PE}$ and postoperative shock. Among all complications, the incidence of GI anastomotic leakage in the AF group was approximately 22 times higher than that in the non-AF group. Although AF may affect the stability of hemodynamics and thus affect the blood supply of the anastomosis, the number of occurrences in both groups is small (5 patients versus 30 ), so further study is needed to confirm the conclusion.

Botwinick et al.[23] reported that patients with AF are more likely to experience gastroparesis, but our study did not find the adverse effect of AF on gastroparesis. There were 249 patients included in Botwinick's study, of which only 13 had atrial fibrillation, so further research may be needed to confirm the relationship between atrial fibrillation and gastroparesis. In addition, no differences were found in GI bleeding, AKI, gastroparesis, blood transfusion, pneumonia, UTI, cardiac arrest, postoperative sepsis, wound complications, etc.

With the advancement of surgical techniques, more and more elderly pancreatic cancer patients with complex comorbidities receive PD. Several studies have shown that age has an important adverse effect on the occurrence of perioperative complications and survival after $\operatorname{PD}[24,25]$. So, we further analyzed patients aged $>65$ years. No significant difference was found in mortality, while there was significant difference between the two groups in LOS and cost. As for complications, the differences in various complications between the AF group and the non-AF group in the elderly population are similar to the overall population. It can be seen that although age will increase complications and mortality, it will increase evenly between the two groups. Further studies are needed to reveal the influence of age on perioperative complications and death after whipple's surgery.

There are several limitations of the present study. First of all, our study is a retrospective study based on the NIS database with all inherent shortcomings. Secondly, because NIS is an inpatient database coding 
with ICD-9-CM, complications without ICD-9 code, such as pancreatic fistula, bile leakage, cannot be analyzed. In addition, tumor information such as tumor type, stage, and neoadjuvant chemotherapy is absent in the NIS database, which may have an impact on the outcomes of OPD. Finally, as the follow-up information is not included within the NIS database, the postoperative outcomes is not discussed in this study. Despite these limitations, this is the first population-based study that investigated the impact of AF on the outcomes of pancreatic cancer patients undergoing OPD, providing surgeons data and serving as a basis for future prospective studies.

\section{Conclusions}

AF increases the cost of pancreatic cancer patients undergoing OPD, also increases the occurrence of cardiac complications, respiratory complications, PE, and postoperative shock. Surgeons should pay special attention to these patients and develop an appropriate preoperative evaluation plan.

\section{Abbreviations}

PD: pancreaticoduodenectomy

OPD: open pancreaticoduodenectomy

AF: atrial fibrillation

NIS: National Inpatient Sample

LOS: length of stay

ICD-9-CM: International Classification of Diseases, Ninth Revision, and Clinical Modification, Ninth Revision

\section{Declarations}

\section{Ethics approval and consent to participate}

This study was exempt from institutional review board evaluation of Beijing Friendship Hospital, Capital Medical University and informed consent was not required because this study was based on the NIS database, which includes fully anonymized and de-identified data.

\section{Consent for publication}

Not applicable.

\section{Availability of data and materials}


The HCUP dataset utilized for this study is publicly available for approved research studies. Further details and instructions for application can be found at: https://www.hcup-us.ahrq.gov/nisoverview.jsp.

\section{Competing interests}

The authors declare that they have no competing interests.

\section{Funding}

This work was supported by National Natural Science Foundation of China (No. 81172318).

\section{Authors' contributions}

$Z Z$ and WG provided administrative support. $\mathrm{PH}$ and $\mathrm{YY}$ contributed to the concept design and the draft of the manuscript. $Y H, H W, D W, K L$ participated in the acquisition of the data. $P H, Y Y, C G$ and $X Z$ conducted statistical analysis. All authors contributed to manuscript writing and approved the final version of the manuscript.

\section{Acknowledgements}

Not applicable.

\section{References}

1. Siegel RL, Miller KD, Jemal A: Cancer statistics, 2020. CA: A Cancer Journal for Clinicians 2020, 70(1):7-30.

2. Fingerhut $A$, Vassiliu $P$, Dervenis $C$, Alexakis $N$, Leandros $E$ : What is in a word: Pancreatoduodenectomy or pancreaticoduodenectomy? Surgery 2007, 142(3):428-429.

3. Kang CM, Lee JH: Pathophysiology after pancreaticoduodenectomy. World Journal of Gastroenterology: WJG 2015, 21(19):5794-5804.

4. T. JC, Samuel WL, S. AJ, Hugh C, E. CJ, C. CJ, B. CJ, T. EP, D. EM, E. FM et al: 2014 AHAVACC/HRS Guideline for the Management of Patients With Atrial Fibrillation: Executive Summary. Circulation 2014, 130(23):2071-2104.

5. Gialdini G, Nearing K, Bhave PD, Bonuccelli U, ladecola C, Healey JS, Kamel H: Perioperative Atrial Fibrillation and the Long-term Risk of Ischemic Stroke. JAMA 2014, 312(6):616-622.

6. Ghani KR, Anson KM, Camm AJ: Atrial fibrillation and the urologist. BJU International 2004, 94(3):254-255.

7. Bargehr J, Trejo-Gutierrez JF, Patel T, Rosser B, Aranda-Michel J, Yataco ML, Taner CB: Preexisting atrial fibrillation and cardiac complications after liver transplantation. Liver Transplantation: Official Publication of the American Association for the Study of Liver Diseases and the International Liver Transplantation Society 2015, 21(3):314-320. 
8. Mohammed S, Fisher WE: Quality Metrics in Pancreatic Surgery. Surgical Clinics of North America 2013, 93(3):693-709.

9. Greenblatt DY, Kelly KJ, Rajamanickam V, Wan Y, Hanson T, Rettammel R, Winslow ER, Cho CS, Weber SM: Preoperative Factors Predict Perioperative Morbidity and Mortality After Pancreaticoduodenectomy. Annals of Surgical Oncology 2011, 18(8):2126-2135.

10. U N, G L, Y G, R G, S P-A, I N, R N, Jm K, N L: Prognostic significance of pancreatic fistula and postoperative complications after pancreaticoduodenectomy in patients with pancreatic ductal adenocarcinoma. The Surgeon: Journal of the Royal Colleges of Surgeons of Edinburgh and Ireland 2019, 18(1):24-30.

11. El Nakeeb A, El Sorogy M, Ezzat H, Said R, El Dosoky M, Abd El Gawad M, Elsabagh AM, El Hanafy E: Predictors of long-term survival after pancreaticoduodenectomy for peri-ampullary adenocarcinoma: A retrospective study of 5-year survivors. Hepatobiliary \& Pancreatic Diseases International 2018, 17(5):443-449.

12. Romano G, Agrusa A, Galia M, Di Buono G, Chianetta D, Sorce V, Gulotta L, Brancatelli G, Gulotta G: Whipple's pancreaticoduodenectomy: Surgical technique and perioperative clinical outcomes in a single center. International Journal of Surgery (London, England) 2015, 21 Suppl 1:S68-71.

13. Karim SAM, Abdulla KS, Abdulkarim QH, Rahim FH: The outcomes and complications of pancreaticoduodenectomy (Whipple procedure): Cross sectional study. International Journal of Surgery 2018, 52:383-387.

14. Saeki H, Tsutsumi S, Tajiri H, Yukaya T, Tsutsumi R, Nishimura S, Nakaji Y, Kudou K, Akiyama S, Kasagi $Y$ et al: Prognostic Significance of Postoperative Complications After Curative Resection for Patients With Esophageal Squamous Cell Carcinoma. Annals of Surgery 2017, 265(3):527-533.

15. Hayashi T, Yoshikawa T, Aoyama T, Hasegawa S, Yamada T, Tsuchida K, Fujikawa H, Sato T, Ogata T, Cho $\mathrm{H}$ et al: Impact of infectious complications on gastric cancer recurrence. Gastric Cancer 2015, 18(2):368-374.

16. Marra F, Steffen T, Kalak N, Warschkow R, Tarantino I, Lange J, Zünd M: Anastomotic leakage as a risk factor for the long-term outcome after curative resection of colon cancer. European Journal of Surgical Oncology (EJSO) 2009, 35(10):1060-1064.

17. Butt JH, Olesen JB, Havers-Borgersen E, Gundlund A, Andersson C, Gislason GH, Torp-Pedersen C, Køber L, Fosbøl EL: Risk of Thromboembolism Associated With Atrial Fibrillation Following Noncardiac Surgery. Journal of the American College of Cardiology 2018, 72(17):2027-2036.

18. Higuchi S, Kabeya Y, Matsushita K, Arai N, Tachibana K, Tanaka R, Kawachi R, Takei H, Suzuki Y, Kogure $\mathrm{M}$ et al: Incidence and complications of perioperative atrial fibrillation after non-cardiac surgery for malignancy. PloS One 2019, 14(5):e0216239.

19. Cameron JL, Riall TS, Coleman J, Belcher KA: One Thousand Consecutive Pancreaticoduodenectomies. Annals of Surgery 2006, 244(1):10-15.

20. Kneuertz PJ, Pitt HA, Bilimoria KY, Smiley JP, Cohen ME, Ko CY, Pawlik TM: Risk of morbidity and mortality following hepato-pancreato-biliary surgery. Journal of Gastrointestinal Surgery: Official 
Journal of the Society for Surgery of the Alimentary Tract 2012, 16(9):1727-1735.

21. V SK, S APJ: Postoperative morbidity following Whipple's procedure for periampullary carcinoma: a retrospective study spanning 5 years. International Journal of Research in Medical Sciences 2019, 7(11):4314-4319.

22. Narayanan S, Martin AN, Turrentine FE, Bauer TW, Adams RB, Zaydfudim VM: Mortality after pancreaticoduodenectomy: assessing early and late causes of patient death. Journal of Surgical Research 2018, 231:304-308.

23. Botwinick IC, Shonkwiler RJ, Steele J, Yu G, Chabot JA: Atrial fibrillation and delayed gastric emptying. PloS One 2011, 6(10):e25499.

24. Sukharamwala P, Prashant S, Thoens J, Jonathan T, Szuchmacher M, Mauricio S, Smith J, James S, DeVito $P$, Peter $D$ : Advanced age is a risk factor for post-operative complications and mortality after a pancreaticoduodenectomy: a meta-analysis and systematic review. HPB: the official journal of the International Hepato Pancreato Biliary Association 2012, 14(10):649-657.

25. Yuan F, Essaji Y, Belley-Cote EP, Gafni A, Latchupatula L, Ruo L, Serrano PE: Postoperative complications in elderly patients following pancreaticoduodenectomy lead to increased postoperative mortality and costs. A retrospective cohort study. International Journal of Surgery 2018, 60:204-209.

\section{Tables}


Table 1

Characteristics of included patients

\begin{tabular}{|llll|}
\hline & No AF & AF & P value \\
\hline Patient characteristics & & & \\
\hline No. (\%) of patients & $11469(90 \%)$ & $1316(10 \%)$ & \\
\hline Female & $5467(47.67 \%)$ & $495(37.64 \%)$ & 0.002 \\
\hline Race & & & 0.023 \\
\hline White & $8806(76.85 \%)$ & $1134(85.48 \%)$ & \\
\hline Black & $1032(9.01 \%)$ & $48(3.63 \%)$ & \\
\hline Hispanic & $845(7.37 \%)$ & $64(4.84 \%)$ & \\
\hline Asian or Pacific Islander & $364(3.17 \%)$ & $32(2.42 \%)$ & \\
\hline Native American & $43(0.37 \%)$ & $0(0 \%)$ & \\
\hline Other & $369(3.23 \%)$ & $48(3.63 \%)$ & \\
\hline Median age, y & 65.1 & 71.9 & $<.274$ \\
\hline Median annual income in patient's zip code, US\$, no. (\%) & & \\
\hline \$1 - \$38,999 & $2416(21.07 \%)$ & $229(17.44 \%)$ & \\
\hline \$39,000 - \$47,999 & $2799(24.40 \%)$ & $326(24.81 \%)$ & \\
\hline \$48,000 - \$62,999 & $2496(21.75 \%)$ & $270(20.53 \%)$ & \\
\hline \$63,000 or more & $2957(25.78 \%)$ & $398(30.23 \%)$ & \\
\hline Insurance type, no. (\%) & $3299(28.75 \%)$ & $362(27.52 \%)$ & \\
\hline Medicaid & & & \\
\hline Medicare & & & \\
\hline Private & & & \\
\hline Uninsured & & & \\
\hline Hospital characteristics & & & \\
\hline Hospital region, no. (\%) & & & \\
\hline Northeast & & & \\
\hline Midwest & & & \\
\hline
\end{tabular}

AF, atrial fibrillation. 


\begin{tabular}{|c|c|c|c|}
\hline & No AF & AF & $P$ value \\
\hline South & $4210(36.70 \%)$ & $480(36.50 \%)$ & \\
\hline West & $2329(20.32 \%)$ & $225(17.11 \%)$ & \\
\hline Hospital bed size, no. (\%) & & & 0.538 \\
\hline Small & $950(8.28 \%)$ & $90(6.84 \%)$ & \\
\hline Medium & $2125(18.53 \%)$ & $270(20.53 \%)$ & \\
\hline Large & $8395(73.19 \%)$ & $955(72.63 \%)$ & \\
\hline Location of hospital & & & 0.444 \\
\hline Rural hospital & $95(0.83 \%)$ & $5(0.38 \%)$ & \\
\hline Urban hospital & 11375 (99.17\%) & $1310(99.62 \%)$ & \\
\hline Teaching status of hospital & & & 0.069 \\
\hline Rural and urban non-teaching hospital & $1140(9.94 \%)$ & $85(6.46 \%)$ & \\
\hline Urban teaching hospital & $10330(90.06 \%)$ & $1230(93.54 \%)$ & \\
\hline \multicolumn{4}{|l|}{ Comorbidity } \\
\hline History of myocardial infarction & $430(3.75 \%)$ & $105(7.98 \%)$ & 0.001 \\
\hline Chronic liver disease & $1385(12.07 \%)$ & $165(12.55 \%)$ & 0.821 \\
\hline Hypertension & $6555(57.15 \%)$ & $935(71.10 \%)$ & $<0.001$ \\
\hline Diabetes mellitus & $3745(32.65 \%)$ & $535(40.68 \%)$ & 0.009 \\
\hline History of stroke & $60(0.52 \%)$ & $20(1.52 \%)$ & 0.038 \\
\hline Peripheral vascular disease & $390(3.40 \%)$ & $90(6.84 \%)$ & 0.004 \\
\hline Obesity & $1190(10.37 \%)$ & $145(11.03 \%)$ & 0.738 \\
\hline Congestive heart failure & $220(1.92 \%)$ & $175(13.31 \%)$ & $<0.001$ \\
\hline Chronic kidney disease & $475(4.14 \%)$ & $125(9.51 \%)$ & $<0.001$ \\
\hline Chronic obstructive pulmonary disease & $810(7.06 \%)$ & $160(12.17 \%)$ & 0.003 \\
\hline Thrombocytopenia & $470(4.10 \%)$ & $65(4.94 \%)$ & 0.516 \\
\hline Valvular heart disease & $290(2.53 \%)$ & $105(7.98 \%)$ & $<0.001$ \\
\hline Coronary artery disease & $1385(12.07 \%)$ & $415(31.56 \%)$ & $<0.001$ \\
\hline Cirrhosis & $147(1.27 \%)$ & $29(2.41 \%)$ & 0.401 \\
\hline
\end{tabular}


Table 2

In-hospital outcomes

\begin{tabular}{|lllll|}
\hline In-Hospital Outcomes & Overall population & \multicolumn{3}{c|}{ Patients $>65$ years } \\
\hline & Adjusted Odds ratio & $p$ Value & Adjusted Odds ratio & p Value \\
\hline Mortality & $3.57(0.86-14.87)$ & 0.080 & $3.35(0.58-19.37)$ & 0.177 \\
\hline Length of stay (day, coefficient) & $0.16(0-0.33)$ & 0.052 & $0.21(0.03-0.39)$ & 0.021 \\
\hline Total cost (US dollar, coefficient) & $0.35(0.06-0.64)$ & 0.020 & $0.38(0.05-0.71)$ & 0.023 \\
\hline GI anastomotic leakage & $22.11(3.25-150.22)$ & 0.002 & NA & NA \\
\hline GI bleeding & $0.54(0.06-4.84)$ & 0.581 & NA & NA \\
\hline AKI & $1.86(0.80-4.29)$ & 0.147 & $1.98(0.87-4.49)$ & 0.102 \\
\hline Gastroparesis & $0.41(0.10-1.61)$ & 0.200 & $0.34(0.05-2.42)$ & 0.284 \\
\hline Blood transfusion & $0.87(0.46-1.64)$ & 0.669 & $0.93(0.45-1.89)$ & 0.831 \\
\hline Cardiac complications & $17.88(5.32-60.11)$ & $<0.001$ & $17.38(3.42-88.43)$ & 0.001 \\
\hline Respiratory complications & $2.38(1.23-4.60)$ & 0.010 & $2.93(1.31-6.53)$ & 0.009 \\
\hline Pneumonia & $0.39(0.07-2.31)$ & 0.299 & $0.13(0.01-1.89)$ & 0.134 \\
\hline PE & $18.53(2.67-128.62)$ & 0.003 & $16.76(0.67-417.50)$ & 0.086 \\
\hline UTI & $1.47(0.65-3.31)$ & 0.353 & $1.65(0.67-4.06)$ & 0.272 \\
\hline Cardiac arrest & $5.50(0.61-49.46)$ & 0.128 & $2.52(0.18-34.92)$ & 0.490 \\
\hline Post-operative sepsis & $0.87(0.29-2.64)$ & 0.808 & $0.89(0.30-2.69)$ & 0.842 \\
\hline Post-operative shock & $5.16(1.03-25.72)$ & 0.045 & $27.06(2.35-311.15)$ & 0.008 \\
\hline Wound complications & $0.70(0.33-1.50)$ & 0.359 & $0.62(0.26-1.49)$ & 0.287 \\
\hline GI, gastrointestinal; AKI, acute kidney injury; PE, pulmonary embolism; UTI, urinary tract infection; \\
\hline
\end{tabular}

\section{Figures}




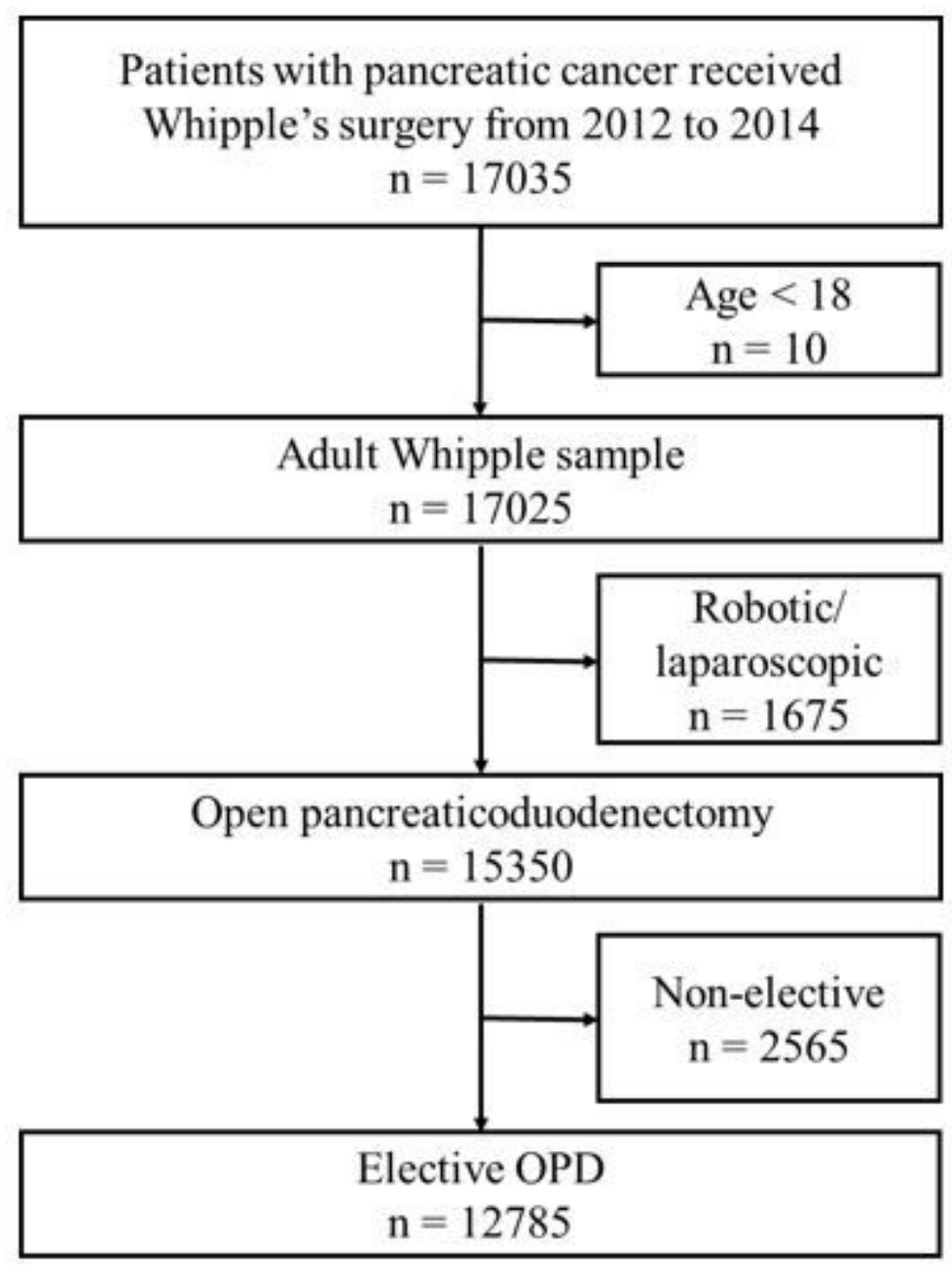

Figure 1

Flow chart of the study population selection process 


\section{Overall population}

Mortality
GI anastomotic leakage
GI bleeding
AKI
Gastroparesis
Blood transfusion
Cardiac complications
Respiratory complication
Pneumonia
PE
UTI
Cardiac arrest
Post-operative sepsis
Post-operative shock
Wound complications

Length of stay (day)

Total cost (US dollar)
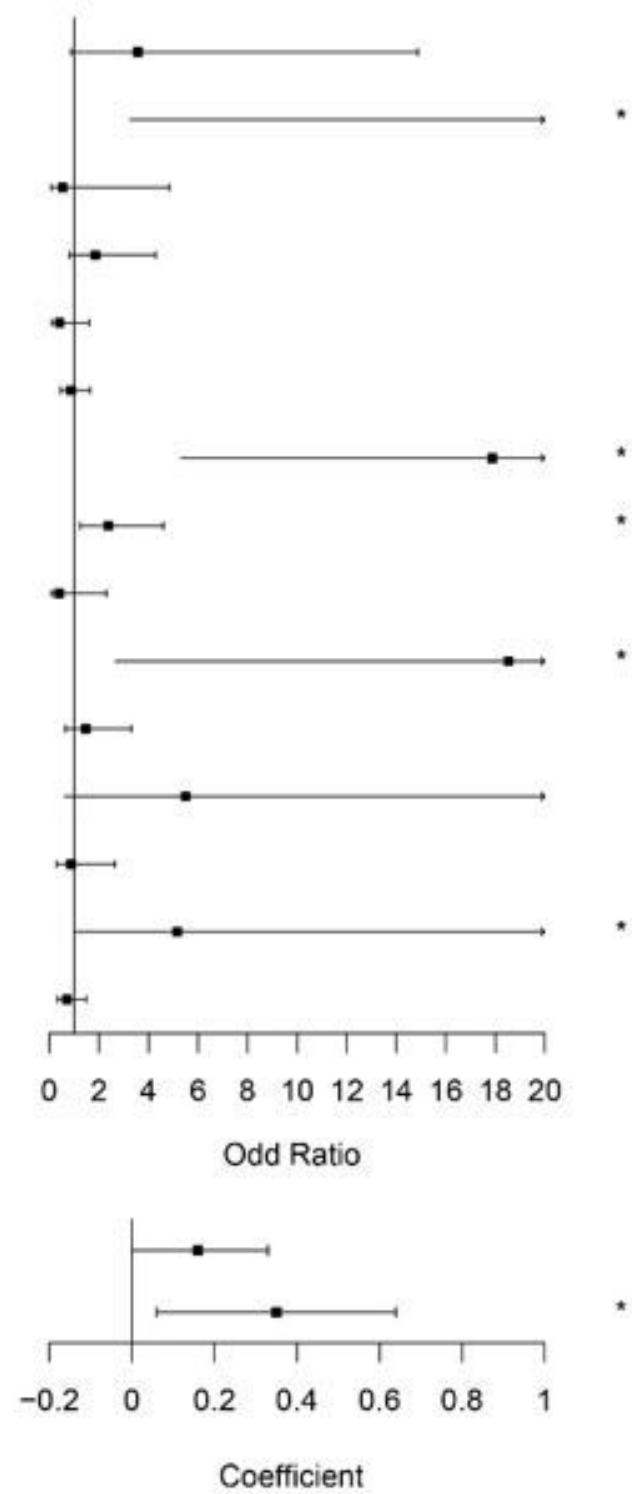

Patients $>65$ years
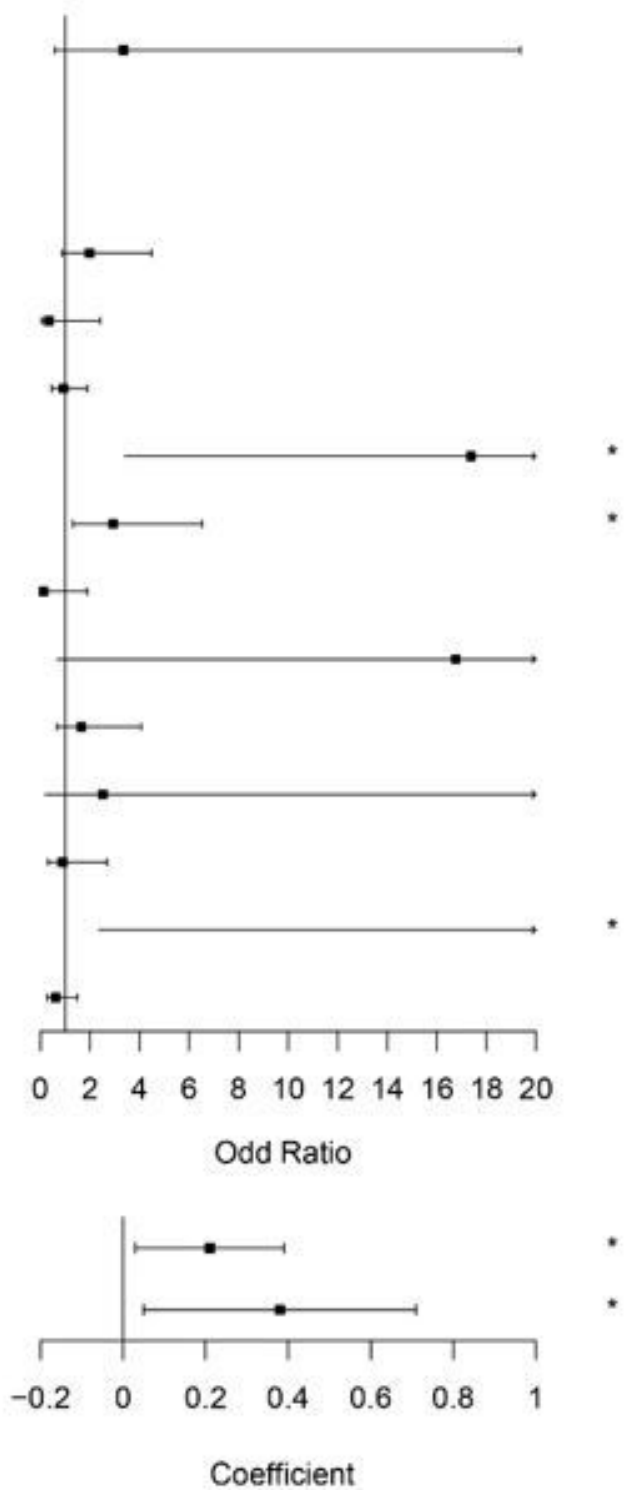

\section{Figure 2}

Forest plot of in-hospital outcomes

\section{Supplementary Files}

This is a list of supplementary files associated with this preprint. Click to download.

- SupplementTable1.docx 